\title{
The effects of rate of exposure upon simultaneous intentional and incidental verbal-discrimination learning'
}

\author{
N. JACK KANAK, DEPARTMENT OF PSYCHOLOGY, THE \\ UNIVERSITY OF OKLAHOMA, Norman, Oklahoma 73069
}

The constant learning-time hypothesis and predictions from frequency theory regarding intentional and incidental verbaldiscrimination learning were tested under three exposure rates. Mean trials and errors were higher under the 1:1 sec rate, but total time was greater with the 4:4 sec rate. Incidental associative recall was greatest within the 4:4 sec rate and was symmetrical under the $2: 2$ and $4: 4 \mathrm{sec}$ rates, but asymmetrical in the $1: 1$ sec condition. The results were explained from an analysis assuming a differential distribution of frequency mechanisms to wrong and right items associated with exposure rate.

Ekstrand, Wallace, \& Underwood (1966) have proposed a frequency theory of verbal-discrimination (VD) learning to account for the processes operative in single-list acquisition of the intentional component (i.e., recognition and overt pronunciation of the right ( $R$ ) item when exposed contiguously with the wrong (W) item) of the task. Kausler (1966), viewing the VD task as a variant of a Type 2 incidental learning design, has presented an analysis of the incidental components which assumes the learning of $\mathrm{W}$ and $\mathrm{R}$ items as responses and the learning of $\mathrm{W}-\mathrm{R}$ and $\mathrm{R}-\mathrm{W}$ pairings as bidirectional associations. Kausler \& Sardello (1967) and Sardello \& Kausler (1967) have shown that these incidental components yield the same functional relationship with pronunciation and degree-of-practice variables as incidental learning in another Type 2 context (Mechanic, 1962). In addition, recent research (Kanak \& Dean, in press) has shown that incidental associative components significantly affect VD transfer, particularly in paradigms involving $R$ item competition.

The present study was designed to determine the sensitivity of VD intentional and incidental learning to variations in rate of exposure of the materials. In VD learning the frequency of representational responses (RRs) and rehearsal of correct responses (RCRs) may be expected to covary positively with increasing exposure time. Intentional learning should benefit from a greater opportunity for RCRs to occur to $\mathrm{R}$ items with a resultant more reliable differential frequency cue. Incidental associative learning should also increase as a function of these RCRs but, in addition, more frequent RRs to both $W$ and $R$ items should result in greater availability of both items for recall. Therefore, the principal hypothesis of the present study was that proficiency of both intentional and incidental learning should increase with increasing exposure time as a function of frequency mechanisms (Ekstrand et al, 1966). In addition, a test of the constant learning-time hypothesis (Bugelski, 1962) will be extended to VD learning and the explanatory power of modifications of that hypothesis (Cooper \& Pantle, 1967) will be examined.

\section{Method}

The design for acquisition was a 2 by 3 factorial with two sets of lists (A and B) combined with three rates of exposure $(1: 1,2: 2$, and 4:4 sec). $W$-R versus $R-W$ associative learning was assessed as a within-S factor via recall tests. The three treatment conditions were randomly assigned to the 48 introductory psychology student volunteers, all naive to verbal learning experiments, in order of their appearance at the laboratory $(\mathrm{N}=16)$.

A pool of 30 associatively unrelated words was selected from the Palermo \& Jenkins (1964) norms. Two subpools of 15 words were then randomly selected to function as $W$ and $R$ items. List $A$ consisted of one set of random pairings of the $W$ and $R$ items; List $B$ was derived by forming new random pairings with $W$ and $R$ item function remaining identical to List $A$. Two lists were utilized to control for possible idiosyncratic pairings of meaningful words. Four different serial orders of W-R pairs, with the left-right secpuence of $W$ and $R$ items varying both within $(8-7$ or $7-8)$ and between serial orders, were prepared for each list to control for serial and spatial position cues.

The materials were presented by the anticipation method on a Lafayette memory drum with a constant 4 sec intertrial interval. The W-R or R-W pairing appeared contiguously in both exposures with the $R$ item underlined in the feedback exposure. Following standard VD instructions focusing on the intentional task, practice was conducted to a criterion of one perfect trial. Following criterion all $S s$ were given a modified free recall (MFR) task without a time limit and with instructions to guess when not certain. The order of MFR was counterbalanced such that half the Ss in each group recalled $W$ itcms to given $R$ items (printed on a sheet of paper) followed by recall of $R$ items to given $W$ items. The remaining Ss had the sequence reversed. Both type of list and order of MFR were unrelated to the acquisition and recall dependent variables as either main effects or components of interactions (all $F$ values yielding $p>.25$ ) and are eliminated from further consideration.

Results

Intentional learning. The mean trials and errors to criterion and the mean total time to learn (seconds per pair) and the corresponding standard deviations for the three conditions are summarized in Table 1 . The analysis of variance indicated significant differences between means for both trials and errors measures, $F(2,45)=12.92$ and $F(2.45)=13.14$, respectively, both ps $<.005$. Duncan's test revealed significant differences on both measures for the $1: 1$ versus $2: 2(\mathrm{p}<.05)$ and $1: 1$ versus $4: 4 \mathrm{sec}$ $(\mathrm{p}<.005)$ comparisons. The $2: 2$ versus $4: 4 \mathrm{sec}$ comparison was not significant on either measure $(p>.10)$.

The test of the constant learning-time hypothesis was conducted on the mean total time per pair for each $S$ (i.e., the product of the number of trials per $S$ multiplied by total exposure time, divided by the number of pairs). The hypothesis was not supported with the between-groups $F(2,45)=11.40, p<.005$. Although heterogeneity of variance was present $\left(F_{\max }=4.70, p<\right.$ $.005)$, an extension of the median test resulted in $\chi^{2}(2)=13.18$, p $<.01$, thus verifying that significant differences in central tendency were indeed present. Total time increased with longer durations of exposure. The differences between the $1: 1$ versus $4: 4$ sec and $2: 2$ versus $4: 4 \mathrm{sec}$ conditions were both highly significant. ps $<.001$ and .005 , respectively. The $1: 1$ versus $2: 2 \mathrm{sec}$ comparison, however, was not significant, $\mathrm{p}>.10$.

Incidental learning. The means and standard deviations for the $W-R$ and $R-W$ directions of recall are presented for each of the experimental conditions in Table 1 . The overall means for direction of recall were $W-R=8.29, R-W=7.56$. The 3 by 2 mixed analysis of variance revealed this within-S difference to be significant, $F(1,45)=7.21, p<.025$, but direction of recall did not significantly interact with rate of exposure, $F(2,45)=1.77$. $p>.10$. The main effect for rate of exposure on overall associative recall was nonsignificant, $F(2,45)=1.89, p>.10$. However, in view of the lack of a significant rate of exposure by direction of recall interaction and the lack of a significant difference between

Table 1

Means and Standard Deviations for Trials and Errors to Criterion, Total Time to Learn, and Bidirectional Associative Recall

\begin{tabular}{|c|c|c|c|c|c|c|}
\hline \multirow[b]{3}{*}{ Variable } & \multicolumn{6}{|c|}{ Treatment Conditions } \\
\hline & \multicolumn{2}{|c|}{$1: 1 \mathrm{sec}$} & \multicolumn{2}{|c|}{$2: 2 \mathrm{sec}$} & \multicolumn{2}{|c|}{$4: 4 \mathrm{sec}$} \\
\hline & Mean & SD & Mean & SD & Mean & SD \\
\hline Trials & 11.38 & 3.86 & 7.44 & 3.57 & 5.63 & 2.04 \\
\hline Ërors & 43.56 & 16.40 & 24.00 & 13.87 & 20.06 & 10.71 \\
\hline Total Time & 22.75 & 7.72 & 29.75 & 14.32 & 45.00 & 16.77 \\
\hline$N-R$ recall & 7.81 & 3.64 & 7.50 & 3.8 .3 & 9.56 & 2.78 \\
\hline$R-W$ recall & 6.44 & 3.20 & 7.38 & $3 . \%$ & 8.87 & 2.85 \\
\hline
\end{tabular}


the $1: 1$ and $2: 2 \mathrm{sec}$ groups on total time to learn, an orthogonal analysis was conducted comparing the $1: 1$ and $2: 2 \mathrm{sec}$ groups with the $4: 4 \mathrm{sec}$ group. The resultant $F(1,45)=7.84$ was significant, $\mathrm{p}<.025$. Thus increasing exposure time did produce a resultant increase in overall associative learning, but not as a linear function with exposure time.

As the means of Table 1 indicate, the significant difference favoring the W-R direction of recall was not of the same magnitude at each rate of exposure. Correlated t-tests $(\mathrm{df}=15)$ indicated that most of the main effect for direction of recall can be attributed to the difference existing within the $1: 1 \mathrm{sec}$ condition, $t=3.34, p<.005$. The comparable tests of the $2: 2$ and $4: 4 \mathrm{sec}$ conditions yielded $\mathrm{t}=.09(\mathrm{p}=.50)$ and $1.64(\mathrm{p}>.05$, one-tail; $>.10$, two-tail), respectively. Thus symmetry of recall was demonstrated for the latter two conditions, though with somewhat less strength for the $4.4 \mathrm{sec}$ condition.

Discussion

The observed patterns of results suggest that total time to learn bears a close relationship to the amount of incidental associative learning and that processes responsible for this relationship may also account for the differences in trials and errors to criterion. The greater total time required by Ss of the $4: 4 \mathrm{sec}$ condition may have been produced by interference effects attributable to a greater frequency of RRs to W items. Cooper \& Pantle (1967) point out that very slow or very rapid rates of presentation may break down the constant learning-time phenomena. Excessive time may result in lapses of study while extremely limited time may impair the production of correct RRs (or RCRs within the Ekstrand et al, framework). Since the VD recognition task is relatively low in difficulty level, the slow 4:4 sec rate may have produced such lapses in study with a resultant increase in attention to irrelevant cues (i.e., the $W$ items) and a concomitant increase in the probability of an incorrect response.

With regard to incidental associative learning, frequency theory (Ekstrand et al, 1966) predicts superior $\mathrm{R}$ item learning and therefore superior $W-R$ recall as a result or more frequent $R R s$, pronunciation responses or PRs, and RCRs to R items. However, the assumed increase in frequency of RRs to $W$ items with increasing exposure time should serve to equalize $W$ and $R$ item availability for recall as well as interfere with intentional learning. These simultaneous effects on intentional and incidental learning are supported by (1) the nonsignificant differences in trials and errors to criterion between the $2: 2$ and $4: 4 \mathrm{sec}$ conditions, and (2) the perfect symmetry of recall by the $2: 2 \mathrm{sec}$ group and the strong statistical tendency toward symmetry within the $4: 4 \mathrm{sec}$ group. To the extent that the 4:4 sec pacing produces increased RCRs as well, the weaker tendency toward symmetry and the superior degree of overall associative learning within this condition are predictable. On the other hand, the $1: 1 \mathrm{sec}$ rate allows little oppor- tunity for RRs to occur to $W$ items with a consequent depression of $W$ item availability and asymmetry of W-R and R-W associative recall. Since the $1: 1$ and $2: 2 \mathrm{sec}$ groups did not differ in total time to learn and in total associative recall, but did differ in trials-errors and the degree of symmetry of recall, it appears that the particular combinations of frequency mechanisms operative during learning are directly related to rate of exposure.

Cooper \& Pantle (1967) suggest that whether or not exposure time is varied factorially is critical to a test of the constant learning-time hypothesis within PA learning. In the VD task, it is conceivable that $\mathrm{RRs}$ to $\mathrm{W}$ items during the anticipation interval might serve to interfere with intentional learning while not greatly facilitating incidental learning, except by increasing item availability. An RR to a $W$ item increases the probability of an incorrect response, but S may not representationally "pair" the items for discrimination learning until the feedback interval. Increases in associative learning may therefore be largely confined to the latter interval. On the other hand, as Cooper and Pantle suggest is true for PA learning, processes operative in the anticipation interval (i.e., RRs) may have the greater effect upon total time to learn.

\section{REFERENCES}

BUGELSKI, B. R. Presentation time, total time, and mediation in pairedassociate learning. J. exp. Psychol., 1962, 63, 409-412.

COOPER, E. H., \& PANTLE, A. J. The total-time hypothesis in verbal learning. Psychol Bull, 1967, 68, 221-234.

EKSTRAND, B. R., WALLACE, W. P., \& UNDERWOOD, B. J. A frequency theory of verbal-discrimination learning. Psychol. Rev., 1966, 6, 566-578.

KANAK, N. J., \& DEAN, M. F. Transfer mechanisms in verbal-discrimination. J. exp. Psychol, in press.

KAUSLER, D. H. Mechanisms for transfer in verbal-discrimination tasks. Invited paper read at the annual meeting of the Southwestern Psychological Association, Arlington, Texas, 1966.

KAUSLER, D. H., \& SARDELLO, R. J. Item recall in verbal-discrimination learning as related to pronunciation and degree of practice. Psychon. Sci., $1967,7,285-286$.

MECHANIC, $A$. The distribution of recalled items in simultaneous intentional and incidental learning. J. exp. Psychol, 1962, 63, 593-600.

PALERMO, D. S., \& JENKINS, J. J. Word association norms: Grade school through college. Minneapolis: University of Minnesota Press, 1964.

SARDELLO, R. J., \& KAUSLER, D. H. Associative recall in verbaldiscrimination learning as related to pronunciation and degree of practice. Psychon. Sci, 1967, 8, 253-254.

\section{NOTE}

1. The author is grateful to Donald H. Kausler who read an earlier draft of the present paper and offered helpful suggestions and to Kenneth McElroy who ran the Ss. The essential results of the study were presented in a paper read at SWPA, New Orleans, 1968. 